\title{
Caracterización y efectividad de programas de rehabilitación neuropsicológica de las funciones ejecutivas en pacientes con daño cerebral adquirido: una revisión*
}

\author{
Neuropsychological Rehabilitation Treatments of Executive Functions in \\ Patients with Brain Damage: Characterization and Effectiveness A Review
}

Recibido: julio 18 de 2013 | Revisado: octubre 23 de 2013 | Aceptado: febrero 27 de 2014

\author{
Adriana Marcela MartíneZ-MartíneZ** \\ Oscar Mauricio Aguilar-Mejía \\ Pontificia Universidad Javeriana, Bogotá, Colombia \\ SUSANA MARTÍNEZ VILLAR *** \\ DANIELA MARIÑo GARCÍA ****
}

doi:10.11144/Javeriana.UPSY13-3.cepr

Parta citar este artículo: Martínez-Martínez, A., A. M., Aguilar-Mejía, O., Martínez, S. \& Mariño, D. (2014). Caracterización y efectividad de programas de rehabilitación neuropsicológica de las funciones ejecutivas en pacientes con daño cerebral adquirido: una revisión. Universitas Psychologica, 13(3),1147-1160. http://dx.doi.org/10.11144/Javeriana.UPSY13-3.cepr

\footnotetext{
* Artículo de revisión.

*** Correos electrónicos: martinez.adriana@javeriana. edu.co,oaguilar@javeriana.edu.co

****martinsusan_2@hotmail.com

*****dmarinogarcia@gmail.com
}

\section{RESUMEN}

En este artículo se realiza una revisión de los principales programas de rehabilitación de las funciones ejecutivas ampliamente reconocidos en la literatura, así como una recopilación de aquellos estudios que han replicado dichos programas en pacientes con daño cerebral adquirido. Estos estudios son seleccionados a través de PubMed al incluir palabras clave tales como: funciones ejecutivas, daño cerebral, evaluación neuropsicológica e intervención. Luego, se analizan a partir de una organización y división de los componentes que trabajan: solución de problemas y memoria de trabajo, autorregulación, motivación e iniciativa y habilidades metacognitivas. Por último se describen aquellos programas que integran más de dos dominios. De la revisión se concluye que existe una gran cantidad de propuestas para la rehabilitación de estas funciones, y queda claro que la rehabilitación de las funciones ejecutivas ofrece efectos positivos sobre el funcionamiento y calidad de vida de la persona, en especial enfocando la intervención a aquellas áreas personales, familiares y ambientales relevantes para su funcionamiento. Sin embargo, aún se carece de suficientes estudios que demuestren la efectividad y eficiencia de dichos programas, lo cual se explica por el uso de muestras pequeñas o de caso único que no permiten la generalización de resultados, las características heterogéneas de los participantes y la baja generalización a la vida cotidiana.

Palabras clave

funciones ejecutivas; rehabilitación neuropsicológica; efectividad

\begin{abstract}
A B S T R A C T
This paper analyses the effectiveness of rehabilitation programs for executive functions in adults with brain damage. We consider an effective treatment when the program shows results with a statistically significant difference in the neuropsychological assessment after intervention $(p<0.05)$. Moreover, others criteria were considered such as improvement on daily life scales, the transfer of strategies on daily life conditions and the persistence of these for at least six months. The articles were searched through PubMed and must include keys words as: executive functions, brain damage, neuopsychological assessment and rehabilitation. Then, they were organised according to which were the aim of the treatment: problem-solving and working memory, self-regulation, motivation and initiative and metacogni-
\end{abstract}


tive skills. Additionally, there is a section where integrative programs were analysed. We concluded that there are many training programs for executive functions with positive results on patients' daily activities when the intervention is focused in metacognitive skills. However, there are still not enough studies that show an accurate effectiveness due to the conceptual complexity of the executive functions, the variability of patients' symptoms and the limited transfer of the knowledge trained to everyday life.

Keywords

executive functions; neuropsychological rehabilitation; effectiveness

\section{Generalidades de las funciones ejecutivas y sus alteraciones}

Luria (1980) a partir del estudio de los cambios cognitivos y comportamentales en pacientes con daño cerebral en áreas frontales del cerebro, fue pionero en plantear que existe una asociación entre ideas, movimientos y acciones simples que se orientan al desarrollo y resolución de conductas complejas. Más adelante, Lezak (1982) retoma los postulados de Luria y plantea el término de funciones ejecutivas definiéndolas como las capacidades necesarias para lograr una conducta eficaz, creativa y socialmente aceptada. Posteriormente, en 1989, Sohlberg y Mateer profundizan en la conceptualización de las funciones ejecutivas, definiéndolas como 'aquellos procesos cognitivos de anticipación, elección de objetivos, planificación, selección de la conducta, autorregulación, autocontrol y retroalimentación'. Estos planteamientos generaron una explosión de investigaciones a partir del estudio de pacientes con lesiones cerebrales que llevaron al desarrollo de teorías que complejizaron aún más dicho concepto. Por ejemplo, Fuster (1989) postula la "teoría de la corteza prefrontal”, en la cual plantea que esta área es fundamental para la estructuración temporal de la conducta. En el mismo sentido, Wilson et al. (1996) citados por Burgess y Robertson (2002), acuñaron el término de síndrome disejecutivo para agrupar una serie de síntomas que suelen aparecer tras lesiones prefrontales, tales como alteración en el pensamiento abstracto, impulsividad, confabulación, dificultades para la planificación, euforia, alteración en la secuenciación temporal, falta de insight, apatía, desinhibición social, motivación fluctuante, agresividad, afecto plano, falta de preocupación, perseveración, inquietud motora, disociación entre saber y hacer, distractibilidad, incapacidad en toma de decisiones y despreocupación por las normas sociales.

Más adelante Lezak, Howieson y Loring (2004) sintetizan las funciones ejecutivas como 'las capacidades del sujeto para ser propositivo, independiente y autorregulado, es decir, lo que le permite saber cuándo, cómo y por qué ejecutar una acción'. Adicionalmente, Cicerone, Levin, Malec, Stuss y Whyte (2006) afirman que el concepto de funciones ejecutivas es "ambiguo" y plantean un esquema a partir de cuatro dominios que facilitan la organización de los programas existentes para la intervención en estas capacidades.

El primer dominio es acerca de "funciones ejecutivas cognitivas" que incluye el control, la dirección de la conducta y la memoria de trabajo. Un segundo dominio implica "funciones de autorregulación conductual". El tercero se centra en "funciones de regulación de la activación", relacionadas con la iniciativa y la motivación. El último dominio incluye "procesos metacognitivos" que se basan en la autoevaluación de las propias habilidades y de la conducta en relación a un objetivo (Cicerone et al., 2006), y se complementa con programas que realizan un abordaje holístico.

A partir de este esquema, se realiza una descripción de los programas de intervención de las funciones ejecutivas que han sido ampliamente reconocidos en la literatura, recopilando aquellos estudios que han replicado dichos programas en pacientes con daño cerebral adquirido. Estos artículos fueron seleccionados a través de PubMed si sus títulos o abstracts incluían palabras clave como: funciones ejecutivas, daño cerebral, intervención y rehabilitación. Los artículos fueron excluidos, si la población de estudio no presentaba daño cerebral adquirido, si incluían muestras infantiles, si estudiaban otras funciones cognitivas como lenguaje o si no incluían resultados de una evaluación neuropsicológica previa a la intervención.

A partir de los estudios encontrados, el presente documento entiende como tratamiento efectivo 
aquel programa que mediante uno o diversos estudios reflejan una diferencia estadísticamente significativa al comparar las puntuaciones de la línea de base y las mediciones posteriores a la intervención entre el grupo de estudio y el grupo control. Por otra parte, se consideran otros criterios de efectividad caracterizados por el aumento de la funcionalidad del paciente, que se refleja en escalas funcionales realizadas posteriormente al tratamiento, la generalización de estrategias a actividades de la vida cotidiana y el mantenimiento de las mismas durante un tiempo igual o mayor a seis meses.

\section{Programas de funciones ejecutivas basados en procesos cognitivos}

Estos programas se basan en el entrenamiento en solución de problemas, en memoria de trabajo y en la creación de ayudas compensatorias para tratar estos déficits. En 1971, D’Zurrilla y Goldfried desarrollan el programa formal de entrenamiento en estrategias para la solución de problemas, el cual consiste en proporcionar a los pacientes estrategias que les permitan reducir la complejidad de un problema, descomponiéndolo en fracciones más simples. Para lograrlo, llevan a cabo una serie de ejercicios en los que la persona debe realizar cada uno de los pasos necesarios para la solución de un problema (identificación del problema, selección de información relevante, procesos de razonamiento, generación de alternativas y toma de decisiones). Von Cramon y Von Cramon (1991, 1992), basándose en la propuesta de D'Zurilla y Goldfried, describen un tratamiento grupal al que llamaron "la terapia de solución de problemas" (PST por sus siglas en inglés: Problem-solving therapy). La PST se estructura en cuatro fases: formulación del problema (definición del objetivo de la tarea), generación de soluciones, selección de la solución más adecuada entre las diversas opciones y verificación del resultado (reconocimiento de errores y corrección).

Para indagar sobre la efectividad del programa propuesto, Von Cramon y Von Cramon (1991) compararon un grupo de pacientes que recibieron la terapia de solución de problemas $(n=20)$ con un grupo de pacientes que participaban en terapia de memoria $(n=17)$; en este último grupo se beneficiaban de sugerencias generales y de actividades grupales, aunque no realizaban tareas ejecutivas específicas. Encontraron que los pacientes del grupo de solución de problemas mostraron una mejoría estadísticamente significativa en la ejecución de pruebas de inteligencia y tareas de funciones ejecutivas; no obstante, no hubo evidencia de generalización a las actividades de la vida diaria.

Rath, Dvorah, Langenbahn, Lynn y Diller (2003) compararon el resultado de 27 pacientes, quienes durante 24 sesiones recibieron un entrenamiento en resolución de problemas basándose en las etapas descritas por Von Cramon y Von Cramon (1991), y 19 pacientes que tomaron un tratamiento que combinaba trabajo psicosocial con tareas cognitivas generales. Encontraron que el grupo de solución de problemas mejoró en el Wisconsin Card Sorting Test, en la autoevaluación de habilidades para solucionar problemas y en la valoración en juegos de roles, mientras que el grupo convencional no mostró cambios. Esta mejoría se mantenía 6 meses después de finalizado el tratamiento.

Robertson (1996) citado por Burgess y Robertson (2002) desarrolló el Goal Management Training (GMT), un método de rehabilitación a partir de la teoría de la negligencia del objetivo de Duncan (1986), el cual consistía en un entrenamiento del manejo de los objetivos. El programa radicaba en que los pacientes identificaran el objetivo principal y los subobjetivos de la tarea, enumerando los pasos necesarios para resolver el problema y posteriormente monitorizar el resultado. El entrenamiento se llevó a cabo en dos sesiones de una hora en sujetos con daño cerebral, seleccionados de forma aleatoria, encontrándose que los pacientes presentaron una disminución estadísticamente significativa en el número de errores cometidos en tareas cotidianas de cocina; adicionalmente, los sujetos reportaban menores dificultades en su vida diaria.

Levine et al. (2000) describieron el uso del GMT en dos estudios; el primero, con un grupo de pacientes con daño cerebral y el segundo, un estudio de caso único con una paciente que había sufrido una meningoencefalitis. En el primer estudio, se encontró que los pacientes mostraban mejoría es- 
tadísticamente significativa en las tareas de papel y lápiz, sin embargo, no se evaluó la generalización a la vida diaria. En el segundo estudio, la paciente tuvo una mejoría estadísticamente significativa en las pruebas post del entrenamiento, pero la ausencia de grupo control pone en duda la generalización de la técnica en otras poblaciones.

Levine et al. (2011) desarrollaron un estudio con 19 pacientes que presentaban daño cerebral focal, en fase de recuperación crónica (más de 6 meses de recuperación), y que por medio de una entrevista reportaban problemas en el funcionamiento ejecutivo. ocho de los participantes hicieron parte del grupo control, mientras que 11 participantes conformaron el grupo GMT (seis pacientes con secuelas de ACV, tres con TCE y uno con tumor frontal). Se administraron 7 sesiones de 2 horas a cada grupo. El grupo control trabajó temas sobre ejercicio físico, nutrición, sueño, comunicación, reducción del estrés, memoria, atención, entre otros temas que no se relacionan directamente con entrenamiento cognitivo de funciones ejecutivas. $\mathrm{La}$ efectividad del tratamiento fue evaluado por medio de una batería de pruebas encargadas de valorar el funcionamiento ejecutivo, que se realizaron pre, post y cuatro meses después de finalizado el tratamiento. Ambos grupos reportaron beneficios de los programas desarrollados, no obstante, el grupo GMT mostró mejoría estadísticamente significativa en la Torre de Londres. Además, mediante una entrevista posterior al tratamiento, se evidenció que los participantes del grupo GMT generalizaron las estrategias aprendidas a actividades de la vida cotidiana.

Novakovic-Agopian et al. (2011) realizan una variante del GMT, en la que se enfatiza en la atención focalizada del "aquí y el ahora" y se trabajan técnicas de relajación. El estudio se llevó a cabo con 16 pacientes con daño cerebral adquirido y un grupo control del mismo tamaño, quienes recibieron educación sobre el daño cerebral adquirido. Aquellos que recibieron la intervención obtuvieron puntuaciones por encima del grupo control en tareas encargadas de evaluar memoria de trabajo, flexibilidad mental, inhibición, atención sostenida, aprendizaje y recuerdo diferido.
Evans (2001) citado por Evans (2009) describe un grupo de tratamiento adaptado desde la teoría de PST y GMT, el cual llamó A\&GM (por sus siglas en inglés: Attention and Goal Management). El objetivo de este grupo era ayudar a las personas a desarrollar una mejor conciencia de sus propias dificultades, al igual que ofrecerles estrategias para manejarlas. Las 12 primeras semanas, se enfoca en la resolución de problemas y manejo de objetivos. En las siguientes semanas, el grupo se reúne una vez por semana durante dos horas, y se trabaja memoria. Además, cada paciente tiene al menos una sesión individual semanal de 40 minutos para revisar el material de las sesiones en grupo, asegurar la comprensión de los temas aprendidos y observar de qué manera se puede aplicar a su cotidianidad.

Miotto, Evans, Souza de Lucia y Scaff (2009) realizaron un estudio que tenía como objetivo investigar la efectividad del grupo A\&GM, con 30 pacientes con daño ejecutivo (16 pacientes con daño frontal izquierdo y 14 con daño frontal derecho). El criterio de inclusión fue tener un déficit en al menos una medida del funcionamiento ejecutivo y que por estas dificultades presentaran una funcionalidad cotidiana significativamente alterada. Se dividieron en tres grupos, teniendo en cuenta que estos quedaran equilibrados en variables como sexo, edad y educación. Inicialmente, el primer grupo recibió el A\&GM, durante 10 semanas; asistían una vez a la semana por 90 minutos; el grupo 2 recibió material que contenía educación e información sobre el daño cerebral, las consecuencias cognitivas, sociales y conductuales y sugerencias sobre ejercicios cognitivos para el hogar. El tercer grupo recibió psicoterapia cuando era necesaria, pero no rehabilitación cognitiva como tal. Seguido del primer periodo todos los pacientes fueron reevaluados. Posteriormente, los grupos 2 y 3 recibieron el tratamiento $A \& G M$ y nuevamente todos los grupos fueron reevaluados. Seis meses después de finalizado el tratamiento, fueron valorados nuevamente. Los autores no encontraron diferencia entre el rendimiento pre y post en las pruebas neuropsicológicas encargadas de evaluar el funcionamiento ejecutivo, y se observó que en varias de las pruebas los tres grupos mostraron mejoría en el primer pe- 
riodo, aunque no fue estadísticamente significativa. No obstante, por medio de escalas funcionales se encontró que los participantes después del $A \& G M$ habían disminuido el número de síntomas disejecutivos, aunque seguían presentando importantes dificultades. Los autores concluyeron que el proceso que más contribuyó al avance en el desarrollo de las actividades diarias fue la estrategia de "parar y pensar", puesto que este proceso permitía el control de la impulsividad, la cual explicaba la mayor parte de sus déficits.

Por otro lado, Shallice y Burgess (1998) a partir de su modelo de solución de problemas asociado al Sistema Atencional Supervisor (SAS), resaltan la importancia de la recuperación de experiencias pasadas, teniendo en cuenta que problemas similares en el pasado pueden ayudar a la situación actual. Hewitt, Evans y Dritschel (2006 citados por Evans, 2009) llevaron a cabo un estudio en el que compararon el rendimiento de dos grupos. A uno se le dio un breve entrenamiento en resaltar experiencias autobiográficas previas en la práctica de solución de problemas actuales, mientras que el otro grupo no recibió entrenamiento. Los resultados mostraron que el grupo que recibió entrenamiento mejoró significativamente en el número de pasos y en la efectividad del plan producido, por lo cual los autores concluyen que la incorporación de alguna forma de entrenamiento relacionado a la recuperación de recuerdos autobiográficos específicos es útil en la solución de problemas cotidianos.

Otro componente cognitivo de las funciones ejecutivas es la memoria de trabajo, la cual repercute de forma significativa en las actividades de la vida cotidiana (Baddeley \& Della, 1998; Baddeley \& Hitch, 1974; Stuss \& Benson, 1984). Duval, Coyette y Seron (2008) desarrollaron un programa de rehabilitación a un paciente que presentaba problemas en el ejecutivo central de la memoria de trabajo. Este se basaba en que algunos pacientes hacen uso de estrategias inefectivas, desarrollando las tareas demasiado rápido y con un inadecuado procesamiento de la información. Para mejorar la rapidez en la ejecución desarrollaron un programa que consistía en una triple estrategia: codificar el material a través del uso del bucle fonológico y la agenda visoespacial, llevar a cabo una organización serial de los procesos mentales y realizar una reducción sistemática en la velocidad de procesamiento. Parten de la hipótesis de que al codificar adecuadamente la información el ejecutivo central no tendrá que usar todos los recursos, dejando algunos libres para el procesamiento de otra tarea. El tratamiento se dividía en dos fases: un programa cognitivo y una rehabilitación ecológica. En el primero, había tres subprogramas: velocidad de procesamiento, codificación de la información (updating) y monitorización de tareas simultáneas. La rehabilitación ecológica tenía dos estados: análisis del escenario y simulación de situaciones de la vida real.

Este programa de rehabilitación se aplicó a un paciente de 23 años que presentaba un déficit selectivo del ejecutivo central. Por medio de la comparación de la evaluación neuropsicológica pre y post, los autores reportan que la rehabilitación fue efectiva para los tres subcomponentes de la memoria de trabajo, refiriendo que además hubo transferencia a las tareas ecológicas. Así mismo, el paciente y sus familiares reportaron mejoría en la ejecución de tareas en la cotidianidad después del programa (Duval et al., 2008).

Bickertona, Humphreysa y Riddocha (2006) realizaron un estudio de caso único en un paciente con un déficit en el ejecutivo central, quien era perseverativo en sus conductas, olvidaba los objetivos de las tareas, fallaba en los pasos para realizar una acción y no era capaz de detectar sus propios errores. La técnica consistió en aprender a verbalizar una actividad diaria en forma de poema, para compensar el déficit de memoria de trabajo mediante el uso de la memoria a largo plazo. El paciente pudo hacer los diferentes pasos de la acción de forma correcta y también corregir sus propios errores, no obstante, con el paso del tiempo el entrenamiento perdió su efectividad. Adicionalmente, se realizó el mismo entrenamiento utilizando la memoria visual, donde el paciente aprendió a relacionar la imagen visual de la tarea con las acciones a desarrollar. Sin embargo, no se encontró generalización a otras tareas de la vida diaria.

En el tratamiento de las funciones ejecutivas cognitivas, también se hace uso de estrategias com- 
pensatorias, como por ejemplo listas de chequeo o libros de notas, en los que el terapeuta enseña al paciente a anotar de forma detallada cada uno de los pasos que se deben realizar para llevar a cabo una actividad determinada (Mateer, 1997). Es fundamental que en este tipo de técnicas el paciente entienda las instrucciones, que se le suministre retroalimentación y asegurarse de que los ejercicios no conlleven ningún peligro (Arango \& Parra, 2008). Von Crammon y Von Cramon (1992) usaron listas de chequeo en un paciente de 33 años, quien debido a un trauma craneoencefálico (TCE) a los 24 años era incapaz de beneficiarse de la retroalimentación, empleaba tiempos prolongados para realizar actividades rutinarias y se le dificultaba afrontar situaciones novedosas, especialmente en su trabajo. Se le elaboró una lista con cada uno de los pasos que debía realizar en su trabajo diario y el paciente debía seguirlos hasta lograr internalizar dicha secuencia, lo cual conllevó a un aumento significativo en el rendimiento laboral del participante; sin embargo, no hubo generalización en nuevas tareas de la vida diaria, por lo que su funcionalidad en otras esferas de la cotidianidad continúo afectada.

Otro estudio relacionado con estrategias compensatorias es el de Manly, Hawkins, Evans, Woldt y Robertson (2002) quienes realizaron una investigación en la que examinaron a 10 pacientes que habían sufrido un TCE un año antes del estudio y quienes experimentaban dificultades en situaciones complejas de la vida cotidiana, porque al realizar una acción finalizaban llevando a cabo otra. Los autores plantean como hipótesis que la ayuda auditiva de un tono durante el desarrollo del Six Elements Test (prueba encargada de evaluar funciones ejecutivas) facilitaría la unión entre el plan del paciente y la acción del mismo. Los investigadores encontraron que el rendimiento de los pacientes fue estadísticamente significativo, mejor cuando estaban expuestos a un tono periódico, pero no predictivo (no había un tono periódico). Lo anterior lo explican planteando que la pista auditiva suspende la actividad actual y permite que evalúen las acciones que están realizando, lo cual aumenta la probabilidad de que el objetivo sea logrado debido a que hay conciencia de este. A partir de los resultados, concluyen que el uso de alertas pueden ser ayudas externas que contribuyen a que los pacientes mejoren su funcionamiento cognitivo en la vida cotidiana.

\section{Programas de funciones ejecutivas basados en autorregulación de la conducta}

Los problemas conductuales de los pacientes con daño cerebral son un obstáculo dentro del proceso de rehabilitación neuropsicológica, debido a que presentan comportamientos de agresividad, impulsividad y desinhibición, los cuales a largo plazo impiden su desarrollo social en el hogar y en la comunidad (Alderman, 1996). Se ha postulado que el uso de estrategias propuestas desde el análisis conductual aplicado (especialmente el reforzamiento diferencial, extinción y tiempo fuera) dentro del proceso de rehabilitación neuropsicológica de las funciones ejecutivas puede disminuir los comportamientos "problema", y de esta forma, influir en la efectividad del programa de rehabilitación cognitiva. El entrenamiento en técnicas de autorregulación y automonitorización conductual tienen como objetivo que los pacientes adquieran la capacidad para supervisar y anticipar las consecuencias de su conducta, regulando su comportamiento y emociones de acuerdo a las demandas ambientales.

Alderman (1996) realizó un estudio con el objetivo de determinar si las diferencias del funcionamiento neuropsicológico se relacionan con el resultado del uso del reforzamiento, extinción y tiempo fuera en el manejo de las alteraciones psicosociales severas que son producto del daño cerebral. En el estudio, se conformaron grupos de 10 participantes divididos en un grupo control y dos grupos de sujetos con daño cerebral. El primer grupo estaba compuesto por pacientes que habían demostrado tener avances conductuales por medio de las técnicas de modificación de conducta (reforzamiento, extinción y tiempo fuera) y en el otro grupo estaban quienes seguían manteniendo las conductas problema a pesar de la intervención comportamental. El autor concluye que los pacientes que no responden a la intervención com- 
portamental tienen una alteración en el "ejecutivo central”, razón por la cual no pueden llevar a cabo dos tareas al mismo tiempo. Afirma que al atender a una tarea dejan a un lado la monitorización de los cambios psicológicos, los pensamientos automáticos y los cambios ambientales, por lo que no hacen uso del feedback ambiental para corregir su conducta. Es así como el reforzamiento o extinción requiere que la persona sea consciente de las contingencias de cualquier programa de reforzamiento, mientras monitorea su propia conducta

Otro tipo de intervenciones se basan en la internalización de estrategias de autorregulación a través del uso de autoinstrucciones verbales (Luria, 1980; Ciceron et al., 2000). En estos programas, el objetivo es que el paciente aprenda a utilizar las autoinstrucciones (lenguaje interno) en actividades de la vida cotidiana. Meichenbaum y Goodman (1971) diseñaron un programa de entrenamiento en autoinstrucciones que comprende cinco pasos basados en los supuestos de Luria, quien refería que el lenguaje posee un papel regulador de la conducta. Los cinco pasos son: el modelado cognitivo, donde el terapeuta realiza la tarea proporcionándose a sí mismo instrucciones en voz alta; la guía externa, en el que el sujeto desarrolla la misma tarea siguiendo las instrucciones dadas por el terapeuta; la autoguía manifiesta, donde el sujeto realiza la tarea dándose instrucciones en voz alta; la autoguía manifiesta atenuada, donde el sujeto ejecuta la tarea susurrando las autoinstrucciones y la autoinstrucción encubierta, en la que el sujeto utiliza el lenguaje interno para guiar su propia conducta. Cicerone y Wood (1987 citados por Evans, 2009), llevaron a cabo un estudio de caso único con un joven de 20 años con daño cerebral severo; el participante era independiente en actividades de la vida diaria, pero presentaba dificultades para inhibir comportamientos inadecuados y ejecutaba acciones sin pensar en sus consecuencias. Se encontró que el paciente mejoró significativamente en aquellas tareas en las que se le entrenó y, además, hubo generalización de la técnica en su conducta social. A partir de este hallazgo se concluyó que dicha técnica permite pensar más lento, lo que a su vez facilita desarrollar el hábito de pensar antes de actuar y no responder de forma impulsiva, influyendo de forma positiva en las actividades de la vida cotidiana.

En síntesis, los estudios disponibles han concluido que las técnicas basadas en el conductismo son efectivas para eliminar o disminuir conductas disruptivas que aparecen 'por exceso' como consecuencia del daño cerebral, pero no cuando su objetivo es implementar un repertorio conductual o mejorar la conducta autoiniciada. Adicionalmente, se ha encontrado que la implementación de estrategias de autorregulación disminuye la impulsividad y contribuye a mejorar la reinserción del paciente en los diferentes ámbitos cotidianos (De Noreña et al., 2010).

\section{Programas de intervención basados en la regulación de la activación}

Estos programas incluyen la iniciativa y la motivación necesaria para desenvolverse en la vida cotidiana, ya que es frecuente encontrar pacientes con problemas para iniciar conductas y/o falta de iniciativa para participar en actividades externas (Gainotti, 1993). A partir de estos planteamientos se han diseñado programas que tienen como objetivo relacionar conductas entre sí, buscando que los pacientes las asocien y que las inicien de forma autónoma (Arango \& Parra, 2008).

Una de las estrategias utilizadas para aumentar la conducta autoiniciada es el uso de ayudas externas. Wilson et al. $(1997,2000)$ citados por Evans (2005) utilizaron un dispositivo conocido como NeuroPage ${ }^{\circledR}$, el cual fue desarrollado por Hersh y Treadgold en 1994, para ayudar a pacientes con problemas de memoria. Evans, Emslie y Wilson (1998) realizaron un estudio de caso único con una paciente que presentaba dificultades para trasladar la intención a la acción, para lo que utilizaron el NeuroPage ${ }^{\circledR}$, como ayuda externa. Encontraron que este fue altamente efectivo para ayudar a la paciente en completar sus tareas en la cotidianidad, evidenciando mejores puntuaciones en escalas de funcionalidad. Se explica la efectividad del programa por dos aspectos: primero, que la presentación 
de un mensaje externo importante para la paciente promovía la realización de la conducta reemplazando la función de la intención interna que fallaba. Segundo, el sonido del sistema activaba el arousal, lo cual facilitaba la iniciación de la tarea y le ayudaba a sostener su atención hasta conseguir el objetivo. Es importante señalar que este tipo de ayuda funciona con conductas simples y muy específicas, como por ejemplo, tomar el medicamento a la hora indicada o acudir a una cita.

Respecto a lo anterior, O'Neill, Moran y Gillespie (2010) señalan que una ventaja de este tipo de herramientas es que proporciona un sentimiento de independencia al paciente, pues no requiere la presencia de sus familiares o cuidadores para llevar a cabo sus actividades; además, es una estrategia más económica que la intervención diaria de un profesional. Por otra parte, concluyen que las ayudas externas como el NeuroPage ${ }^{\circledR}$ serían mucho más efectivas si la ayuda fuese verbal y estuviese proporcionada por sonidos familiares como la voz de los cuidadores; así, la atención no se fijaría solo en el aspecto físico de la herramienta y proporcionaría una mayor familiaridad para el paciente.

Otra estrategia compensatoria que es utilizada para mejorar los problemas de iniciativa es el "scaffolding" (andamiaje), el cual se basa en la guía de otra persona para iniciar y finalizar una tarea. Stone (1998) llevó a cabo un estudio haciendo uso de guías verbales a ocho pacientes con demencia, quienes presentaban importantes dificultades para iniciar conductas de la vida cotidiana, tales como utilizar una prótesis. Se encontró que seis de ellos lograron realizar la actividad sin ayuda, después del entrenamiento mediante andamiaje.

A partir de lo anterior, se puede concluir que la rehabilitación de síntomas relacionados con una disminución en la iniciativa o la motivación requiere una implementación rigurosa de estrategias externas que contribuyan con el sujeto a autoiniciar su conducta; no obstante, aún son muy pocos los estudios que describen y evidencian la efectividad de estrategias internas para la intervención en la falta de iniciativa.

\section{Programas de intervención basados en funciones metacognitivas}

En los programas de rehabilitación neuropsicológica es imprescindible abordar la conciencia del déficit para obtener resultados significativos en el paciente (Burgess \& Robertson, 2002; Fleming $\&$ Ownsworth, 2006). Fleming y Ownsworth (2006) señalan que la conciencia no es un constructo único sino que hay grados de afectación. Adicionalmente, plantean que la conciencia implica diferentes procesos neurales cognitivos y psicológicos, por lo que es necesario distinguir entre una falta de conciencia como secuela de daño cerebral y la negación del déficit como un mecanismo psicológico.

Dentro de las principales técnicas generadas para tratar el déficit de conciencia, se encuentra el suministro de información al paciente acerca de la naturaleza de la lesión, explicar cómo el daño cerebral afecta su comportamiento y las razones por las cuales varios de los comportamientos que exhibe no son apropiados (Mateer, 1999). Así mismo, es fundamental que el proceso de intervención cuente con soporte emocional a los pacientes y familiares, con el objetivo de que logren manejar adecuadamente la conciencia del déficit y el impacto del mismo sobre su vida cotidiana (Fleming, Lucas \& Lightbody, 2006).

Ownsworth, McFarland y Young (2000) realizaron una investigación en la que estudiaron tres variables: las mejorías en la autoconciencia, en las habilidades de autorregulación y en el funcionamiento psicosocial. Se realizaron talleres informativos a los pacientes en los que se hacía una introducción del aspecto que se iba a trabajar, luego el grupo definía los conceptos tratados y hablaban acerca de las dificultades en esta área y las estrategias que utilizaban para afrontarlos. Los autores encontraron, mediante escalas funcionales y observación estructurada, efectos positivos sobre las relaciones sociales y un aumento de la autoconciencia del déficit, al explicar sus dificultades. No obstante, refieren que aquellos pacientes que hacían uso de la negación como un mecanismo psicológico, no mostraban mejoría en el funcionamiento psicosocial. 
Desde otro enfoque, Toglia y Kirk (2000) exponen el modelo comprensivo, interaccional y dinámico de la conciencia, el cual se basa en la dinámica de la autoconciencia más que en la arquitectura. Proponen el uso del "dominio guiado", en el que se involucra al paciente en experiencias estructuradas que permitan la autoevaluación y el automonitoreo. Las tareas pueden ser acompañadas con preguntas y con grabaciones para ofrecer una posterior autoevaluación. Los autores proponen una serie de estrategias que permiten mejorar la autoconciencia como son la anticipación de obstáculos de la actividad, autopredicción del nivel de dificultad, automonitoreo y estimación del tiempo y posibilidad de éxito. Otra técnica es la inversión de roles en la que el terapeuta realiza la tarea cometiendo errores y el paciente debe identificarlos y nombrarlos. LandaGonzalez (2001) demostró la efectividad del modelo de Toglia y Kirk con un estudio de caso único, de un paciente con trauma craneoencefálico quien por medio de un entrenamiento de ocho horas por semana durante seis meses, logró incorporar las estrategias aprendidas en un múltiple número de escenarios de la vida diaria, mejorando su desempeño en estas situaciones.

En otro estudio de Fleming y Ownsworth (2006), intervinieron sobre cuatro pacientes con daño cerebral, realizando un programa de 10 semanas con actividades basadas en el modelo de Toglia y Kirk, en el que los participantes mostraron ganancias en el nivel de autoconciencia, pero al mismo tiempo hubo aumento en el estrés emocional reportado en escalas funcionales y entrevistas.

Por otra parte, Burgess, en 1996 (citado en Burgess \& Robertson, 2002), desarrolló un programa basado en ayudas externas para estudiar pacientes con daño cerebral adquirido, en el cual introducía claves auditivas como ayuda externa para el control del tiempo, lo cual llevaba a que los pacientes aumentaran el nivel de automonitorización de sus desempeños y por ende a disminuir de forma significativa el número de errores cometidos. Este cambio le permitió concluir que el mejorar el soporte ambiental para el desarrollo de tareas que implican funciones ejecutivas, puede facilitar la monitorización y la flexibilidad conductual.
Se puede concluir, tal y como lo evidencian Cicerone et al. (2000) y Cicerone et al. (2005), que es fundamental que todo programa de rehabilitación de las funciones ejecutivas esté acompañado de estrategias que permitan generar un adecuado nivel de conciencia del déficit, lo que permitirá que el paciente logre automonitorizar su desempeño y crear estrategias para disminuir el impacto de las secuelas cognitivas sobre su funcionalidad. Teniendo en cuenta la importancia de este componente en el proceso de rehabilitación, es necesario encaminar las investigaciones a la creación de estrategias efectivas que permitan alcanzar dicho objetivo.

\section{Programas multidimensionales de intervención en funciones ejecutivas}

Teniendo en cuenta la gran complejidad de las funciones ejecutivas, algunos autores han creado diferentes programas que abordan simultáneamente distintas áreas de esta función. Entre estos se encuentran Rath et al. (2003), quienes proponen un tratamiento para los déficits en solución de problemas en pacientes con traumatismo craneoencefálico, el cual se centra en la autorregulación emocional y el razonamiento lógico. Se basan en la idea de que la solución de problemas está compuesta por dos aspectos: el primero, las habilidades hacia el problema, que incluye definirlo y formularlo, generar alternativas, tomar una decisión, implementar la solución y verificar la obtención del resultado esperado. El segundo componente es la orientación hacia el problema, que quiere decir la actitud y motivación hacia este; para tratar este aspecto se facilita la motivación y el sentimiento de autoeficacia. La novedad del tratamiento fue incluir el aprendizaje de la automonitorización emocional en las tareas de orientación hacia la solución de problemas. Se les enseñó a buscar señales tanto de expresión como de arousal que van a predecir y registrar una conducta impulsiva no adaptativa mediante la práctica de juego de roles y entrevistas individuales. A partir de esto se plantearon estrategias alternativas para no realizar esas conductas. Aquellos que recibieron el programa integrado mostraron mejores resultados 
en las pruebas y escalas encargadas de resolución de problemas y automonitorización emocional. Se evidenció generalización en la vida diaria, lo cual se atribuye al elemento facilitador para el desarrollo de las relaciones sociales, a partir de los juegos de roles realizados.

Partiendo del enfoque anteriormente descrito, Rath, Hradil, Litke y Diller (2011) resaltan que es crucial tener en cuenta los factores psicológicos de los pacientes, para que un programa de rehabilitación cognitiva sea efectivo. Expresan la importancia de desarrollar estrategias de autorregulación emocional que permitan tratar las creencias maladaptativas que desarrollan los pacientes sobre sus capacidades cognitivas y que influyen negativamente en la rehabilitación de capacidades cognitivas. De esta forma, toda intervención neuropsicológica debe tener en cuenta el componente motivacional, emocional y actitudinal, para maximizar la efectividad de cualquier programa.

Los programas descritos en este apartado evidencian que las intervenciones basadas en varias dimensiones de las funciones ejecutivas permiten una rehabilitación integral que tiene en cuenta los diversos factores psicosociales con influencia sobre el paciente y su familia, permitiendo un mayor impacto en el funcionamiento de la vida cotidiana.

\section{Conclusiones}

Las funciones ejecutivas se han definido como "las capacidades necesarias para que un individuo en su vida diaria, logre una conducta eficaz, creativa y socialmente aceptada" (Lezak, 1982). Este constructo implica una compleja e intrincada relación entre los procesos particulares de aprendizaje, las demandas del entorno y el desarrollo del sistema nervioso central (especialmente de la corteza prefrontal). El anterior análisis de los diferentes programas de rehabilitación neuropsicológica de las funciones ejecutivas permite concluir que la efectividad de la intervención se sustenta en la implementación de estrategias adecuadas para la resolución de problemas y su aplicación a situaciones de la vida diaria en contextos reales y diversos, mediante una práctica repetida y el uso de reforzamiento.
Igualmente, muestra que la efectividad se refleja en el aumento de las puntuaciones en pruebas neuropsicológicas específicas, lo cual se corrobora en el estudio realizados por Kennedy et al. (2008), en el que reporta que la mayoría de estudios encuentran resultados positivos inmediatamente después del tratamiento. También, cabe resaltar que el mantenimiento y la generalización de los programas utilizados en la rehabilitación de las funciones ejecutivas dependen de la implementación de estrategias de automonitorización y autoevaluación y del grado de autoconciencia del paciente, lo cual potencia los resultados del proceso terapéutico (Cicerone et al., 2000; Cicerone et al., 2005; Kennedy et al., 2008).

Sin embargo, como lo muestran los diferentes modelos teóricos, la adquisición de las funciones ejecutivas involucra un alto grado de variabilidad y complejidad que no puede abarcarse en su totalidad con un único y sencillo programa de intervención neuropsicológica, principalmente por la variedad de patologías neurológicas que producen un déficit en funciones ejecutivas y por la gran diversidad de déficits cognitivos, emocionales y conductuales asociados a las lesiones prefrontales, y que además están mediados por el contexto donde se desarrolla el sujeto. Por todo lo anterior, las diferentes investigaciones señalan claramente que no existe un programa único que sea efectivo en todos los pacientes con déficit en funciones ejecutivas, sino que estos se deben establecer de forma particular y de acuerdo a las características clínicas y psicosociales de cada sujeto.

El análisis de los diferentes estudios permite determinar que son pocas las investigaciones centradas en determinar la efectividad de los programas de intervención, especialmente por las limitaciones metodológicas para extrapolar y generalizar los resultados de estudios con muestras pequeñas o de caso único (Wall, Turner \& Clarke, 2013); así mismo, el análisis de la efectividad de los programas se afecta porque las características de los participantes son heterogéneas, y con frecuencia los logros de una intervención en un espacio terapéutico o de investigación no se pueden generalizar a contextos como el trabajo o el hogar (Kennedy et al., 2008). Igualmente, existen un número limitado de estu- 
dios que realicen un seguimiento de las funciones ejecutivas a largo plazo, teniendo en cuenta que los beneficios últimos del tratamiento dependerán de la continuidad de los sujetos para hacer uso de las estrategias en situaciones cotidianas. De esta forma, no solo es importante realizar una evaluación a largo plazo, sino también continuar con el soporte e intervención después del periodo inicial del tratamiento.

Por otra parte, se evidencia que la mayoría de los programas planteados para la rehabilitación de las funciones ejecutivas se centran en un solo componente de este amplio constructo (funciones cognitivas ejecutivas, funciones de autorregulación, funciones de regulación de la activación o funciones metacognitivas), siendo pocos los programas que buscan un abordaje terapéutico que abarque varias dimensiones y pocos estudios enfocados en probar la efectividad de los programas para mejorar la regulación de la activación (iniciativa y motivación) y de las técnicas conductuales aplicadas al campo clínico de la neuropsicología, siendo fundamental consolidar el conocimiento científico sobre estos temas, ya que juegan un papel fundamental en el nivel de funcionalidad de los pacientes.

Para finalizar, después de analizar las fortalezas y debilidades de los diferentes estudios, se pueden puntualizar ciertas recomendaciones que permitirán construir un programa de rehabilitación óptimo para cada paciente. Queda claro que la rehabilitación de las funciones ejecutivas ofrece efectos positivos sobre el funcionamiento de la persona (Calleo et al., 2012; Kluwe-Schiavon, Sanvicente-Vieira, Kristensen \& Grassi-Oliveira, 2013). El terapeuta, al plantear un programa de intervención, debe basarse en el resultado de la evaluación neuropsicológica en la que se identifican las fortalezas y debilidades del paciente, adecuando la intervención a aquellas áreas personales, familiares y ambientales relevantes para su funcionamiento. La efectividad del programa no solo dependerá de la técnica utilizada, sino de los esfuerzos realizados por el terapeuta (actitud, compromiso y capacidad para proveer un contexto en el que el individuo pueda tener éxito) y por el paciente, siendo indispensable que este último sea capaz de asumir la responsabilidad que implica toda la rehabilitación neuropsicológica (Cicerone et al., 2000; Ylvisaker \& Feeney, 1998).

De esta forma, se puede asumir que el análisis exhaustivo de todas las variables implicadas y la integración de técnicas de restauración, compensación y modificación ambiental son una buena opción para garantizar la efectividad del programa. Además, es fundamental que la intervención elegida se ajuste a las características del individuo y que esta se modifique o se mantenga en función de su progreso (Goldstein, Levin, Boake \& Lohrey, 1990; Levin, 1989; Levin \& Goldstein, 1986; SchmitterEdgecombe, Marks, Fahy \& Long, 1992; Ylvisaker \& Feeney, 1998).

Así mismo, se debe tener claro que el éxito del programa no solo dependerá de las técnicas utilizadas, sino que en principio todos los terapeutas deben partir de la idea de que las funciones ejecutivas están compuestas por cuatro grandes dominios (cognitivo, autorregulación, activación y metacognición) y, por lo tanto, deben ser trabajados de forma conjunta. De acuerdo con esta conclusión, también se recomienda que se incorporen intervenciones psicológicas para problemas de control emocional y habilidades psicosociales en los programas de funciones ejecutivas.

Para finalizar, toda intervención neuropsicológica deberá medir su efectividad no solo a través de medidas objetivas, sino también por medio de escalas de funcionalidad en la vida cotidiana y una observación conductual estructurada en diferentes escenarios que permitan evaluar cualitativamente los logros alcanzados (Lewis, Babbage \& Leathem, 2011). Además, es importante evaluar los resultados con base en la integración del individuo a la comunidad y la percepción de su calidad de vida (Cicerone et al., 2000; Cicerone et al., 2005). En lo concerniente a investigaciones futuras, teniendo en cuenta la dificultad de llevar a cabo la comparación entre los diferentes tratamientos, y por ende, una evaluación de una intervención, se debe mejorar la especificación de las bases teóricas, integrando diferentes aproximaciones a la rehabilitación cognitiva (Stuss, 2011). A nivel metodológico, se debe hacer uso de diferentes diseños que permitan identificar y discriminar los componentes necesarios para una 
intervención efectiva. Adicionalmente, es necesario replicar intervenciones que hayan reportado ser efectivas, para incrementar la predicción de los tratamientos y comparar diferentes técnicas para distintos déficits (Cicerone et al., 2005). A partir de lo anterior, se puede concluir que un largo número de investigaciones han demostrado que la rehabilitación cognitiva es efectiva, no obstante, aún es necesario seguir investigando su efectividad y eficacia, además de plantear preguntas específicas sobre aspectos puntuales del proceso terapéutico y las características del paciente, para optimizar los resultados clínicos de la intervención.

\section{Referencias}

Alderman, N. (1996). Central executive deficit and response to operant conditioning methods. Neuropsychological Rehabilitation, 6(3), 161-186.

Arango, J. \& Parra, M. (2008). Rehabilitación de las funciones ejecutivas en caso de patología cerebral. Neuropsicología, Neuropsiquiatría y Neurociencias, 8(1), 159-178.

Baddeley, A. \& Della Sala, S. (1998). Working memory and executive control. En A. C. Robert, T. W. Robbins \& L. Weiskrantz (Eds.), The prefrontal cortex: Executive and cognitive functions (pp. 9-21). New York: Oxford University Press.

Baddeley, A. D. \& Hitch, G. J. (1974). Working memory. En G. A. Bower (Ed.), The psychology of learning and motivation: Advances in research and theory (Vol. 8, pp. 47-90). New York: Academic Press.

Calleo, J., Burrows, C., Levin, H., Marsh, L., Lai, E. \& York, M. (2012). Cognitive rehabilitation for executive dysfunction in Parkinson's Disease: Application and current directions. Parkinson's Disease, Artículo ID 512892. Disponible en http://dx.doi. org/10.1155/2012/512892

Bickertona, W., Humphreysa, G. \& Riddocha, J. (2006). The use of memorised verbal scripts in the rehabilitation of action disorganisation syndrome. Neuropsychological Rehabilitation, 16(2), 155-177.

Burgess, P. \& Robertson, I. (2002). Rehabilitation of frontal lobe function. En D. Stuss \& R. Knight (Eds.), Principles of frontal lobe function (pp. 557570). New York: Oxford University Press.
Cicerone, K., Dahlberg, C., Kalmar, K., Langenbahn, D., Malec, J., Bergquist, T., Felicetti, T., et al. (2000). Evidence-based cognitive rehabilitation: Recommendations for clinical practice. Archives of Physical Medicine and Rehabilitation, 81(12), 1596-1615.

Cicerone, K., Dahlberg, C., Malec, J., Langenbahn, D., Felicetti, T., Kneipp, S., Ellmo, W., et al. (2005). Evidence-based cognitive rehabilitation: Updated review of the literature from 1998 through 2002. Archives of Physical Medicine and Rehabilitation, 86(8), 1681-1692.

Cicerone, K., Levin, H., Malec, J., Stuss, D. \& Whyte, J. (2006). Cognitive rehabilitation interventions for executive function: Moving from bench to bedside in patients with Traumatic Brain Injury. Journal of Cognitive Neuroscience, 18(7), 1212-1222.

D’Zurrilla, T. J. \& Goldfried, M. R. (1971). Problemsolving and behavior modification. Journal Abnormal Psychology, 78, 107-126.

De Noreña, D., Sánchez, I., García, A., Tirapu, J., Bombin, I. \& Ríos, M. (2010). Efectividad de la rehabilitación en el daño cerebral adquirido (II): funciones ejecutivas, modificación de conducta y psicoterapia, y uso de nuevas tecnologías. Revista de Neurología, 51(12), 733-744.

Duncan, J. (1986). Disorganisation of behaviour after frontal lobe damage. Cognitive Neuropsychology, 3(3), 271-290.

Duval, J., Coyette, F. \& Seron, X. (2008). Rehabilitation of the central executive component of working memory: A re-organisation approach applied to a single case. Neuropsychological Rehabilitation, 18(4), 430-460.

Evans, J. (2005). Can be executive impairments effectively treated? En P. Halligan \& D. Wade (Eds.), The effectiveness of rehabilitation for cognitive deficits (pp. 247-256). Oxford: Oxford University Press.

Evans, J. (2009). The cognitive group, part 1: Attention and goal management. En B. Wilson, F. Gracey, J. Evans, J. \& A. Baterman (Eds.), Neuropsychological rehabilitation. Theory, models, therapy and outcome (pp. 81-97). Cambridge: Cambridge University Press.

Evans, J., Emslien, H. \& Wilson, B. (1998). External cueing systems in the rehabilitation of executive 
impairments of action. Journal of the International Neuropsychological Society, 4(4), 399-408.

Fleming, J. M., Lucas, S. E. \& Lightbody, S. (2006). Using occupation to facilitate selfawareness in people who have acquired brain injury. Canadian Journal of Occupational Therapy, 73(1), 44-55.

Fleming, J. M. \& Ownsworth, T. (2006). A review of awareness interventions in brain injury rehabilitation. Neuropsychological Rehabilitation, 16(4), 474-500.

Fuster, J. M. (1989). The prefrontal cortex: Anatomy, physiology and neuropsychology of the frontal lobe (2.a ed.). New York: Raven Press.

Gainotti, G. (1993). Emotional and psychosocial problems after brain injury. Neuropsychological Rehabilitation: An International Journal, 3(3), 259-277.

Goldstein, F. C., Levin, H. S., Boake, C. \& Lohrey, J. H. (1990). Facilitation of memory performance through induced semantic processing in survivors of severe closed-head injury. Journal of Clinical and Experimental Neuropsychology, 12(2), 286-300.

Kennedy, M., Coelho, C., Turkstra, L., Ylvisaker, M., Moore Sohlberg, M., Yorkston, K., Chiou, H., et al. (2008). Intervention for executive functions after traumatic brain injury: A systematic review, meta-analysis and clinical recommendations. Neuropsychological Rehabilitation, 18(3), 257-299.

Kluwe-Schiavon, B., Sanvicente-Vieira, B., Kristensen, C. \& Grassi-Oliveira, R. (2013). Executive functions rehabilitation for schizophrenia: A critical systematic review. Journal of Psychiatric Research, 47(1), 91-104.

Landa-Gonzalez, B. (2001). Multicontextual occupational therapy intervention: A case study of traumatic brain injury. Occupational Therapy International, 8(1), 49-62.

Levin, H. S. (1989). Memory deficit after closed-head injury. Journal of Clinical and Experimental Neuropsychology, 12(1), 129-153.

Levin, H. S. \& Goldstein, F. C. (1986). Organization of verbal memory after severe closed-head injury. Journal of Clinical and Experimental Neuropsychology, 8(6), 643-656.

Levine, B., Robertson, I. H., Clare, L., Carte, G., Hong, J., Wilson, B., Duncan, J., et al. (2000). Rehabilitation of executive functioning: An experimental- clinical validation of goal management training. Journal of the International Neuropsychological Society, 6(3), 299-312.

Levine, B., Schweizer, T., O'Connor, C., Turner, G., Gillingham, S., Stuss, D., Manly, T., et al. (2011, 17 de febrero). Rehabilitation of executive functioning in patients with frontal lobe brain damage with goal management training. Frontiers in Human Neuroscience, 5, Artículo 9.

Lewis, M., Babbage, D. \& Leathem, J. (2011). Assessing executive performance during cognitive rehabilitation. Neuropsychological Rehabilitation: An International Journal, 2(2), 145-163.

Lezak, M. D. (1982). The problem of assessing executive functions. International Journal of Psychology, 17(2-3), 281-297.

Lezak, M., Howieson, D. \& Loring, D. (2004). Neuropsychological assessment. New York: Oxford Press.

Luria, A. R. (1980). Lenguaje y comportamiento. Barcelona: Fontanella.

Manly, T., Hawkins, K., Evans, J., Woldt, K. \& Robertson, I. (2002). Rehabilitation of executive function: Facilitation of effective goal management on complex tasks using periodic auditory alerts. Neuropsychologia, 40(3), 271-281.

Mateer, C. A. (1997). Rehabilitation of individuals with frontal lobe impairment. En J. Leon-Carrion (Ed.), Neuropsychological rehabilitation: Fundamentals, innovations and directions (pp. 285-300). Delray Beach, FL: GR Press/St. Luice Press.

Mateer, C. A. (1999). The rehabilitation of executive disorders. En D. T. Stuss, G. Winocur \& I. Robertson (Eds.), Cognitive neurorehabilitation (pp. 314-332). Cambridge: Cambridge University Press.

Meichenbaum, D. H. \& Goodman, J. (1971). Training impulsive children to talk to themselves: A means of developing self-control. Journal of Abnormal Psychology, 77(2), 115-126.

Miotto, E., Evans, J., Souza de Lucia, M. \& Scaff, M. (2009). Rehabilitation of executive dysfunction: A controlled trial of an attention and problem solving treatment group. Neuropsychological Rehabilitation, 19(4), 517-540.

Novakovic-Agopian T., Chen A. J., Rome S., Abrams, G., Castelli, H., Rossi, A., McKim, R., et al. (2011). Rehabilitation of executive functioning with train- 


\section{Adriana Marcela Martínez Martínez, Oscar Mauricio Aguilar Mejía, Susana Martínez Villar, Daniela Mariño García}

ing in attention regulation applied to individually defined goals: A pilot study bridging theory, assessment, and treatment. Journal of Head Trauma Rehabilitation, 26(5), 325-338.

O’Neill, B., Moran, K. \& Gillespie, A. (2010). Scaffolding rehabilitation behaviour using a voice mediated assistive technology for cognition. Neuropsychological Rehabilitation, 20(4), 509-527.

Ownsworth, T., McFarland, K. \& Young, R. McD. (2000). Self-awareness and psychosocial functioning following acquired brain injury: An evaluation of a group support programme. Neuropsychological Rehabilitation, 10(5), 465-484.

Rath, J., Dvorah, S., Langenbahn, D., Lynn, R. \& Diller, L. (2003). Group treatment of problem-solving deficits in outpatients with traumatic brain injury: A randomised outcome study. Neuropsychological Rehabilitation, 13(4), 461-488.

Rath, J., Hradil, A., Litke, D. \& Diller, L. (2011). Clinical applications of problem-solving research in neuropsychological rehabilitation: Addressing the subjective experience of cognitive deficits in outpatients with acquired brain injury. Rehabilitation Psychology, 56(4), 320-328.

Schmitter-Edgecombe, M. E., Marks, W., Fahy, J. F. \& Long, C. J. (1992). Effects of severe closed-head injury on three stages of information processing. Journal of Clinical and Experimental Neuropsychology, 14(5), 717-737.

Shallice, T. \& Burgess, P. (1998). The domain of supervisory processes and the temporal organization of behaviour. En A. C. Robert, T. W. Robbins \& L. Weiskrantz (Eds.), The prefrontal cortex: Executive and cognitive functions (pp. 22-33). New York: Oxford University Press.

Sohlberg, M. M. \& Mateer, C. A. (1989). Introduction to cognitive rehabilitation. New York: Guilford Press.

Stone, C. A. (1998). The metaphor of scaffolding: Its utility for the field of learning disabilities. Journal of Learning Disabilities. 331, 344-364.

Stuss, D. T. (2011). The future of cognitive neurorehabilitation. Neuropsychological Rehabilitation: An International Journal, 21(5), 755-768.

Stuss, D. T. \& Benson, D. F. (1984). Neuropsychological studies of the frontal lobes. Psychological Bulletin, 95(1), 3-28.

Toglia, J. \& Kirk, U. (2000). Understanding awareness deficits following brain injury. NeuroRehabilitation, 15(1), 57-70.

Von Cramon, D. \& Von Cramon, G. (1991). Problem solving deficit in brain injuried patients: A therapeutic approach. Neuropsychological Rehabilitation, 1(1), 45-64.

Von Cramon, D. \& Von Cramon, G. (1992). Reflections on the treatment of brain injured patients suffering from problem-solving disorders. Neuropsychological Rehabilitation, 2(3), 207-230.

Wall, G., Turner, A. \& Clarke, R. (2013). Evaluation of neuropsychological rehabilitation following severe traumatic brain injury: A case report. Neurocase: The Neural Basis of Cognition, 19(6), 530-541. http://dx.doi.org/10.1080/13554794.2012.701642

Ylvisaker, M. \& Feeney, T. J. (1998). Collaborative brain injury intervention: Positive everyday routines. San Diego, CA: Singular Publishing Group Inc. 